OPEN ACCESS

Edited by:

Jia Huang,

Tongji University, China

Reviewed by:

Weiguo Huang,

Fujian Institute of Research on the

Structure of Matter (CAS), China

Guoqing $Z u$,

Tongji University, China

Jiaqing Zhao,

Shanghai Aerospace Electronic

Technology Research Institute, China Yuanyuan $\mathrm{Hu}$,

Hunan University, China

*Correspondence:

Wenwu Li

wwli@ee.ecnu.edu.cn

Specialty section:

This article was submitted to Energy Materials,

a section of the journal

Frontiers in Materials

Received: 26 March 2020

Accepted: 20 April 2020

Published: 14 May 2020

Citation:

Xu Y, Huang F and Li W (2020)

Schottky-Barrier-Dependent Electrical

Characteristics in Conjugated

Polymer Transistors With Various

Contact Metals. Front. Mater. 7:131.

doi: 10.3389/fmats.2020.00131

\section{Schottky-Barrier-Dependent Electrical Characteristics in Conjugated Polymer Transistors With Various Contact Metals}

\author{
Yang Xu, Fanming Huang and Wenwu Li* \\ Engineering Research Center of Nanophotonics and Advanced Instrument (MOE), Technical Center for Multifunctional \\ Magneto-Optical Spectroscopy (Shanghai), School of Physics and Electronic Science, East China Normal University, \\ Shanghai, China
}

Despite the intensive study on the promotion of device performance, the device physics regarding the effects of Schottky barrier on the charge injection in conjugated polymer transistors still need more discussions. Here, the indacenodithiophene-cobenzothiadiazole (IDT-BT) organic field-effect transistors (OFETs) with four different contact metals ( $\mathrm{Pt}, \mathrm{Au}, \mathrm{Cu}$, and $\mathrm{Cr}$ ) were fabricated to explore the effects. Different Schottky barriers were achieved with the contact electrode's work function $\left(W_{F}\right)$ varying. The $I_{\text {ON }} / I_{\text {OFF }}$ ratio of IDT-BT OFETs increases from $10^{3}$ to $10^{6}$ as the $W_{F}$ increases from 4.6 to $5.65 \mathrm{eV}$. The hole mobility also increases from 0.01 (for $\mathrm{Cr}$ ) to $2.79 \mathrm{~cm}^{2} \mathrm{~V}^{-1} \mathrm{~s}^{-1}$ (for $\mathrm{Pt}$ ) when the $W_{F}$ approaches the highest occupied molecular orbital (HOMO) level of the conjugated polymer. Moreover, the threshold voltage and subthreshold swing of the devices both decrease with increasing the $W_{F}$. These could be ascribed to the lowered Schottky barrier with $W_{F}$, which promotes charge injection. The lowest Schottky barrier $(0.123 \mathrm{eV})$ and contact resistance $\left(1.06 \times 10^{4} \Omega \cdot \mathrm{cm}\right)$ can be achieved in the devices with Pt contacts, owing to the highest work function.

Keywords: charge injection, OFETs, Schottky barrier, mobility, contact resistance

\section{INTRODUCTION}

Solution-processed organic field-effect transistors (OFETs) are promising for next-generation consumer electronics, because of the unique advantages of low cost, flexibility, and large-area fabrications (Liu et al., 2010; Jiang et al., 2019). In order to improve the device performance for applications, much efforts have been paid to the material synthesis, film microstructure, device fabrication, and charge transport optimization of OFETs (Chen et al., 2012; Yuan et al., 2014; Uemura et al., 2016). Among these researches, charge injection, which occurs at the interface between metal and organic semiconductor (OSC), is one of the key factors to promote the device performance. Due to the presence of the Schottky barrier, charge injection can be significantly limited. According to the Schottky-Mott limit, the Schottky barrier height can be roughly estimated from the difference between the contact electrode's work function $\left(W_{F}\right)$ and the transporting energy level of the OSC (Tang et al., 2016).

On the other hand, the contact resistance $\left(R_{C}\right)$ of OFETs is very high, which is due to the absence of doping as well as the heterogeneous materials at the electrode-semiconductor junction 
(Liu et al., 2015). With the channel length reducing, the $R_{C}$ impact is amplified, further degrading the charge injection (Choi et al., 2018; Wang et al., 2018). Therefore, the relationship between the charge injection and the device characteristics needs to be investigated in detail. The $W_{F}$ of these four contact metals is $5.65,5.10,4.65$, and $4.60 \mathrm{eV}$, respectively. So different heights of Schottky barriers can be formed in IDT-BT OFETs, which will help us to systematically explore the Schottky-barrier-dependent electrical characteristics.

In this work, the Schottky barrier, the RC, and charge injection in indacenodithiophene-co-benzothiadiazole (IDT-BT) OFETs with four different contact metals ( $\mathrm{Pt}, \mathrm{Au}, \mathrm{Cu}$, and $\mathrm{Cr}$ ) were studied. The effects of the Schottky barrier on the performance parameters such as the mobility, the threshold voltage $\left(V_{T H}\right)$, and subthreshold swing (SS) have also been explored.

\section{DEVICE FABRICATION AND CHARACTERIZATIONS}

The device structure of the top-gate staggered IDT-BT transistors and the molecular structure of IDT-BT polymer are shown in Figures 1A,B. The drain and source electrodes of different metals ( $\mathrm{Pt}, \mathrm{Au}, \mathrm{Cu}$, and $\mathrm{Cr}$ ) with thickness of $30 \mathrm{~nm}$ were made on pre-cleaned Corning glass substrate by magnetron sputtering through shadow mask. The channel width $(W)$ was fixed to $1200 \mu \mathrm{m}$ while the channel length $(L)$ varies from 60 to $350 \mu \mathrm{m}$. The conjugated polymer, IDT-BT, dissolved in dichlorobenzene $(8 \mathrm{mg} / \mathrm{mL})$ was spin-coated at $2000 \mathrm{r} / \mathrm{min}$ in argon-fill glove box and then annealed in the same glove box at $100^{\circ} \mathrm{C}$ for $1 \mathrm{~h}$. Subsequently, polystyrene dissolved in butyl acetate $(80 \mathrm{mg} / \mathrm{mL})$ was spin-coated at $2000 \mathrm{r} / \mathrm{min}$ as the gate dielectric and then annealed at $80^{\circ} \mathrm{C}$ for $12 \mathrm{~h}$. Finally, the top gate electrodes of $\mathrm{Al}(80 \mathrm{~nm})$ were prepared by thermal evaporation through shadow mask. The thicknesses of the IDTBT semiconductor and PS dielectric layer were estimated to about 50 and $1000 \mathrm{~nm}$, respectively. The electrical properties of the fabricated IDT-BT OFETs were characterized at the temperature of 100-280 K by using probe station (Lakeshore TTPX) equipped with semiconductor parameter analyzer (Keithley 4200-SCS).

\section{RESULTS AND DISCUSSION}

\section{On-Off Ratio Analysis}

Figures 2A,B show the typical saturation and linear transfer characteristics of the devices with four different contact metals (where $W / L=1200 / 60 \mu \mathrm{m}$ ). In the saturation regime (at the drain
A

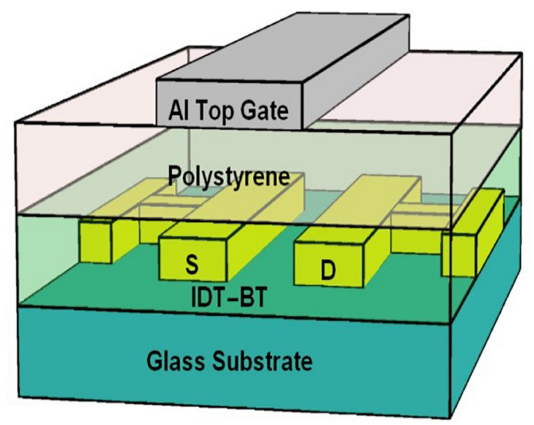

B

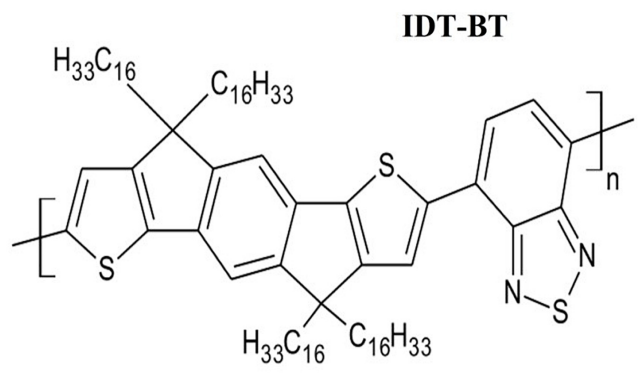

FIGURE 1 | (A) Schematic illustration of the device structure of top-gated IDT-BT OFETs. (B) Molecular structure of IDT-BT polymer.
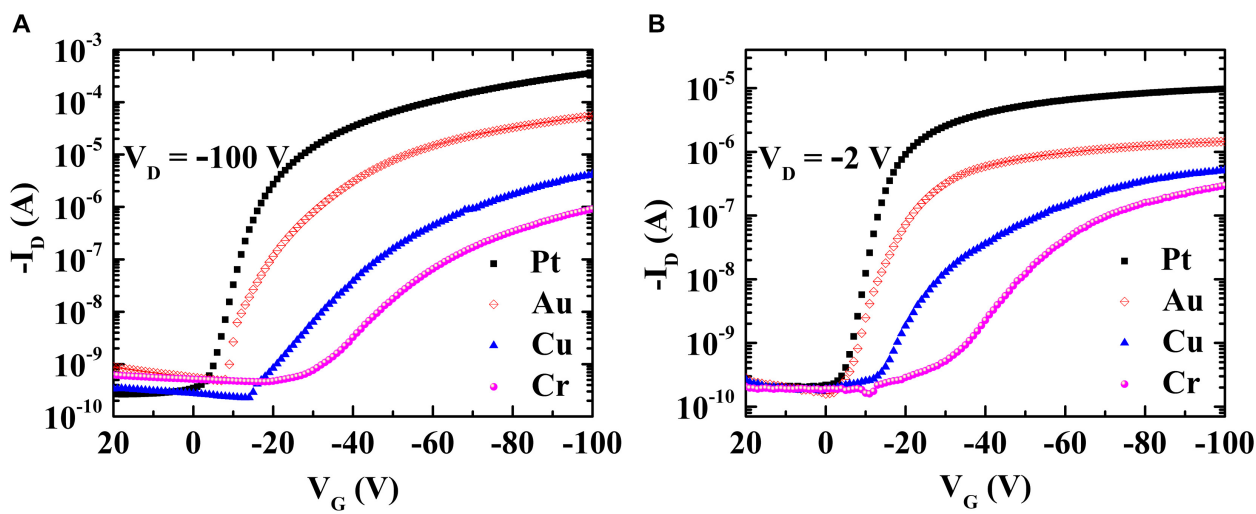

FIGURE 2 | (A) Saturation and (B) linear transfer curves of the IDT-BT OFETs with four different contact metals Pt, Au, Cu, and Cr, respectively. 
TABLE 1 | The key electrical parameters of IDT-BT OFETs with four different contact metals.

\begin{tabular}{|c|c|c|c|c|c|c|}
\hline Metal & $W_{F}(\mathrm{eV})$ & $I_{O N} / I_{O F F}$ & $\mu_{S A T}\left(\mathrm{~cm}^{2} \mathrm{~V}^{-1} \mathrm{~s}^{-1}\right)$ & $V_{T H}(\mathrm{~V})$ & SS $\left(\operatorname{Vdec}^{-1}\right)$ & $R_{C}(\Omega \cdot \mathrm{cm})$ \\
\hline $\mathrm{Pt}$ & 5.65 & $1 \times 10^{6}$ & 2.79 & -18.41 & 2.14 & $1.06 \times 10^{4}$ \\
\hline $\mathrm{Au}$ & 5.10 & $2 \times 10^{5}$ & 1.20 & -29.17 & 3.83 & $1.03 \times 10^{5}$ \\
\hline $\mathrm{Cu}$ & 4.65 & $1 \times 10^{4}$ & 0.03 & -31.87 & 7.70 & $3.86 \times 10^{6}$ \\
\hline $\mathrm{Cr}$ & 4.60 & $2 \times 10^{3}$ & 0.01 & -49.79 & 11.71 & $4.37 \times 10^{6}$ \\
\hline
\end{tabular}

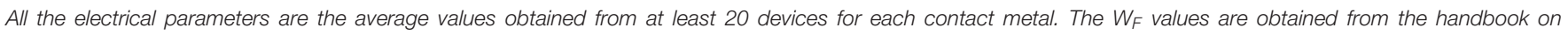
metal work function.

voltage $\left.V_{D}=-100 \mathrm{~V}\right)$, the $I_{O N} / I_{O F F}$ ratio constantly increases from $10^{3}$ to $10^{4}, 10^{5}$, and $10^{6}$ for the transistors with $\mathrm{Cr}, \mathrm{Cu}$, $\mathrm{Au}$, and $\mathrm{Pt}$ contacted, respectively, mainly due to the rising on-state drain current $\left(I_{D}\right)$ that augments from $10^{-6} \mathrm{~A}(\mathrm{Cr}$ contact) to $10^{-4} \mathrm{~A}$ (Pt contact). The result is consistent with the lower Schottky barrier for higher work-function metal adopted in the OFETs, as predicted by the Schottky-Mott limit where the Schottky barrier equals to the energy difference between the metal's work function $\left(W_{F}\right)$ and the highest occupied molecular orbital (HOMO) level of the OSC.

\section{Key Electrical Parameters of Devices}

The key electrical parameters are summarized in Table 1. The rising on-state current suggests the promotion of the mobility. The saturation field-effect mobility $\left(\mu_{S A T}\right)$ is thus calculated from the saturation regime by using the following equation:

$$
\mu_{S A T}=\frac{2 L}{W} C_{I N S} \cdot\left(\frac{\partial\left(I_{D}\right)^{1 / 2}}{\partial V_{G}}\right)^{2}
$$

where $L, W, I_{D}, V_{G}$, and $C_{I N S}$ are the channel length, channel width, drain current, gate voltage, and unit-area gate capacitance of the gate dielectric, respectively (Uemura et al., 2016). As listed in Table 1, the $\mu_{S A T}$ constantly increases from 0.01 to $0.03,1.20$, and $2.79 \mathrm{~cm}^{2} \mathrm{~V}^{-1} \mathrm{~s}^{-1}$ for the OFETs with $\mathrm{Cr}, \mathrm{Cu}$, $\mathrm{Au}$, and Pt contacts, respectively. The IDT-BT OFETs with Pt electrodes show the highest mobility $\left(2.79 \mathrm{~cm}^{2} \mathrm{~V}^{-1} \mathrm{~s}^{-1}\right)$. It is two times higher than the mobility of IDT-BT OFETs with Au electrodes (Shin et al., 2017; Wang et al., 2017). This value is even higher than the devices with $\mathrm{Cu}$ and $\mathrm{Cr}$ electrodes by two orders of magnitude. This result indicates that the contact metals with different $W_{F}$ can indeed significantly affect the device performance by altering charge injection. The examination of the literature shows that the $W_{F}$ of $\mathrm{Pt}$ is $5.65 \mathrm{eV}$, which is nearest to the HOMO level of IDTBT $(5.4 \mathrm{eV})$ (Xu et al., 2017). Hence, the Schottky barrier for $\mathrm{Pt}$ contact OFETs is $0.25 \mathrm{eV}$, which is beneficial for the hole injection from the electrode to the channel. For the other three metals, the value is at least $0.3 \mathrm{eV}$. The energy barriers are really high, so the hole injection from the source electrodes into channel is difficult. Therefore, the charge transport and the electrical performance of the OFETs are deteriorated.

The sub-threshold swing (SS) and the threshold voltage $\left(V_{T H}\right)$ reflect the influence of the Schottky barrier (Jiang et al., 2019). This is because the charge injection can also be limited by high
Schottky barrier that is not directly or not efficiently modulated by the gate voltage. Thus, it leads to slow and late charge accumulation, i.e., large SS and high $V_{T H}$. Note that small SS and $V_{T H}$ are desirable for practical applications especially for high-speed operation and low-power consumption (Lai et al., 2018; Wei et al., 2018). From Table 1, we can find that the SS and $V_{T H}$ values monotonically decrease with increasing the contact's $W_{F}$, indicating the improved charge injection. Furthermore, the electrical parameters can be modulated with different heights of Schottky barrier. High Schottky barrier was formed, when the $W_{F}$ difference between the contact metal and the HOMO level of IDT-BT is large. It will further lead to poor charge injection and non-ohmic contacts. On the other hand, a nearly ohmic contact is expected, when the $W_{F}$ of the contact metal approaches the HOMO level of IDTBT. It will promote the charge injection and accumulation (Ishii et al., 1999).

\section{Effect of Contact Metals on Contact Resistance}

Due to the absence of doping in OFETs, the contacts between metal and OSC are basically Schottky contacts with great barrier height. The contact resistance $\left(R_{C}\right)$ is usually very high, which is a critical issue to OFETs (Liu et al., 2015). The transfer line method (TLM) can be adopted to extract the $R c$ of the IDT-BT OFETs. In

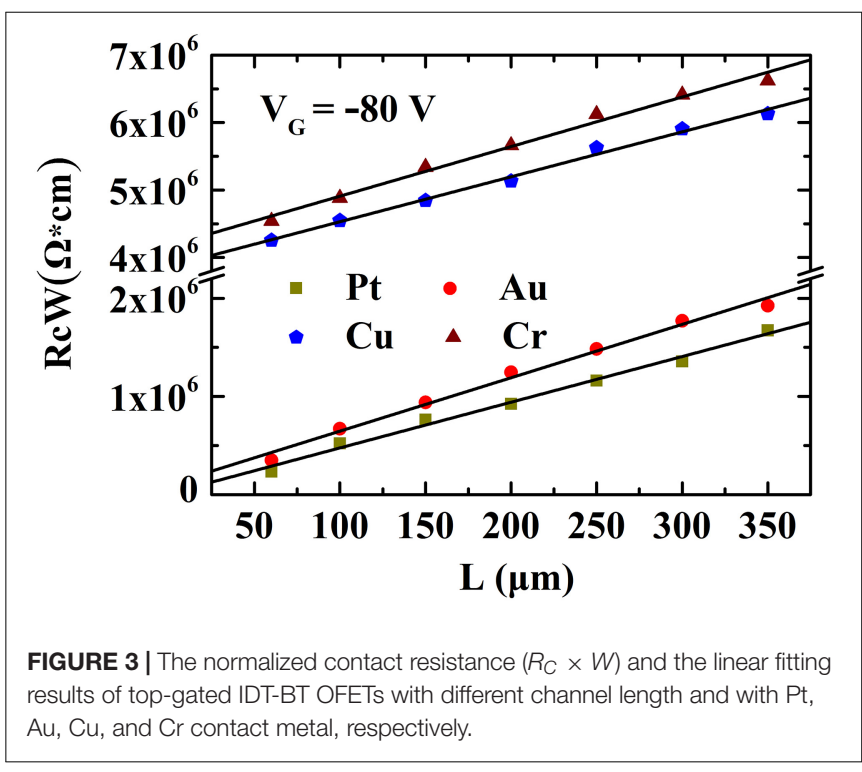



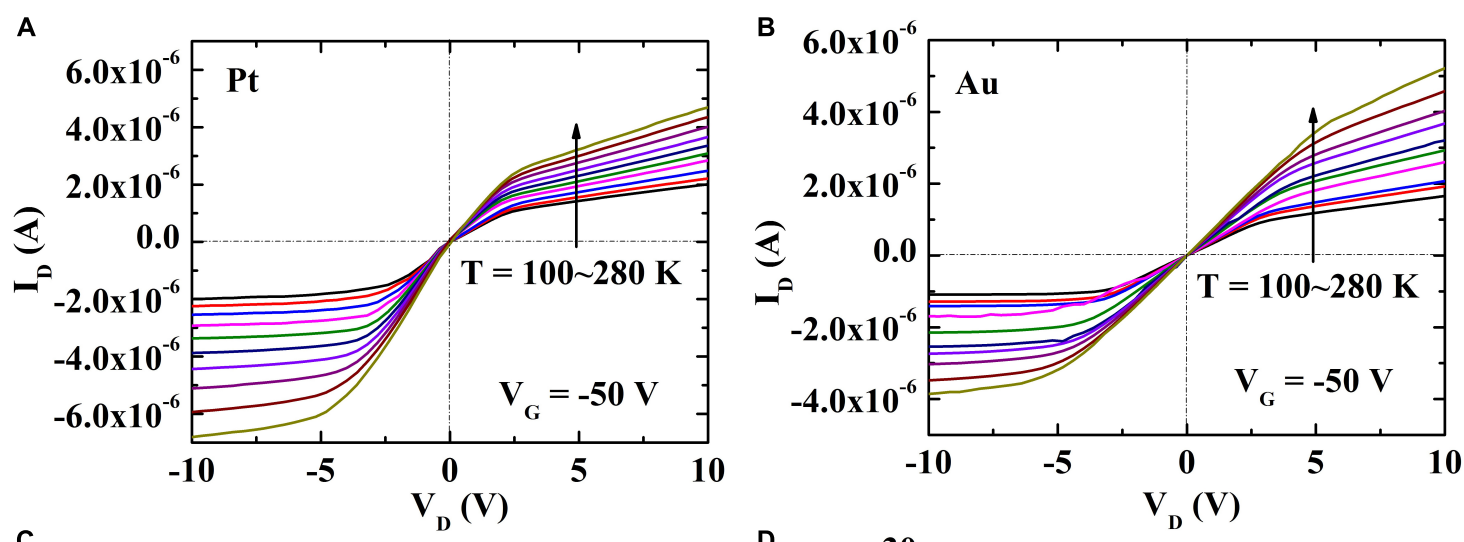

C

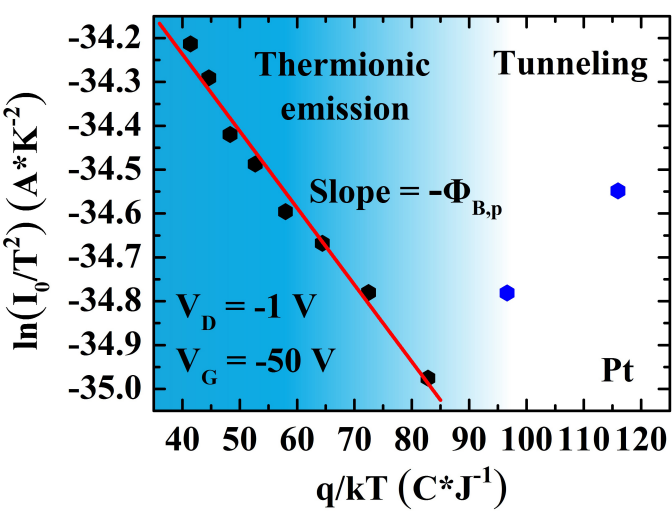

E

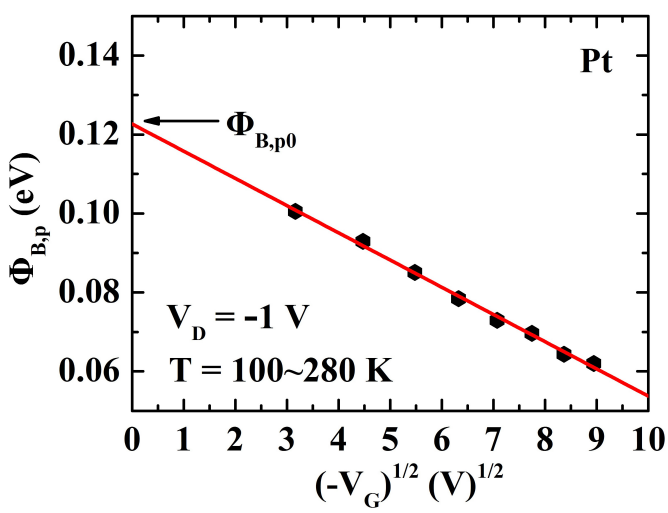

D

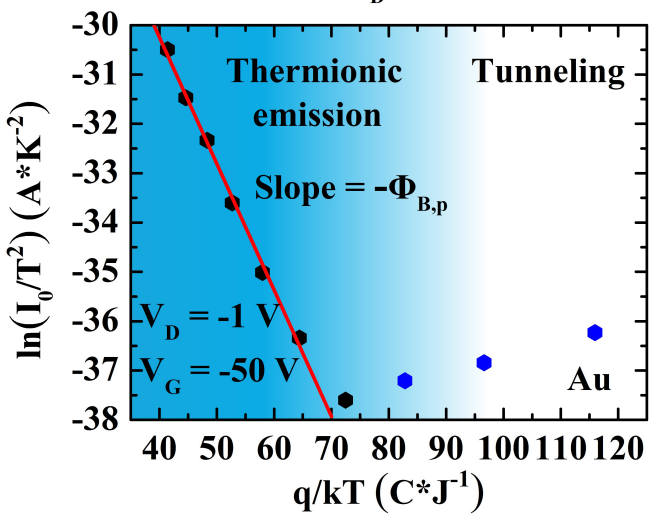

$\mathbf{F}$

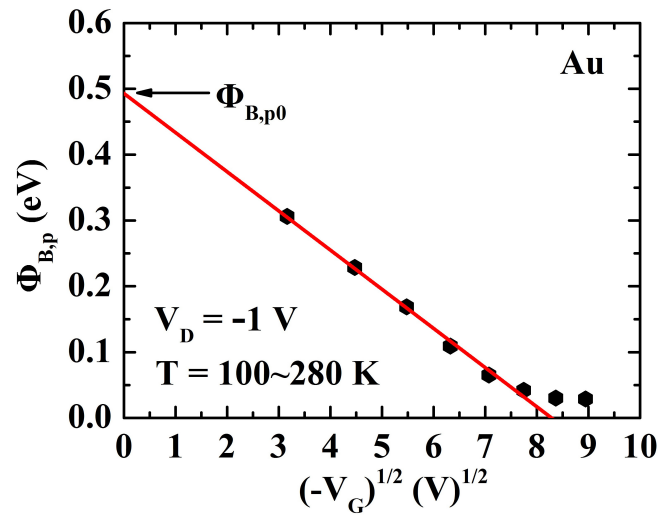

FIGURE 4 | Output characteristics of top-gated IDT-BT OFETs with (A) Pt and (B) Au contacts at the temperatures varying from 100 to $280 \mathrm{~K}$; linear fit for the thermionic emission model for the devices with (C) Pt and (D) Au contacts. The Schottky barrier $\left(\Phi_{B}\right)$ is extracted from the slope of the linear fitting of $\ln \left(l_{0} / T^{2}\right)$ versus $(q / k T)$ curve; linear fit for the image-force barrier lowering arising from $V_{G}$ for the devices with $(\mathbf{E}) \mathrm{Pt}$ and $\mathbf{( F )}$ Au contacts. An intrinsic barrier $\left(\Phi_{B, p 0}\right)$ is obtained by the intercept at zero $V_{G}$, where $V_{D}=-1 \mathrm{~V}$.

the linear regime, the total resistance $\left(R_{T O T}\right)$ can be written as the sum of the $R_{C}$ and channel contact $\left(R_{C H}\right)$ according to:

$$
R_{T O T}=R_{C}+R_{C H}=\frac{V_{D}}{I_{D}}
$$

where $V_{D}$ is the drain voltage. The $R_{C}$ is independent on the channel length $(L)$, while the $R_{C H}$ proportionally declines with reducing $L$. Therefore, for a fixed $V_{G}$, when $R_{T O T}$ is plotted against $L$, a straight line can be obtained with whose $y$-axis intercept is $R_{C}$ (Natali and Caironi, 2012). For our devices, the contact resistance normalized by the channel width $\left(R_{C} \times W\right)$ is shown in Figure 3. Note that the $R_{C}$ is clearly lower for the $\mathrm{Au}$ and $\mathrm{Pt}$ contacted OFETs, compared to $\mathrm{Cu}$ and $\mathrm{Cr}$ contacted devices. A lowest value of $1.06 \times 10^{4} \Omega \cdot \mathrm{cm}$ is obtained for Pt-based devices at the $V_{G}$ of $-80 \mathrm{~V}$. The $R_{C}$ increases to $1.03 \times 10^{5} \Omega \cdot \mathrm{cm}$ for the OFETs with Au electrodes, and then markedly increases to $3.86 \times 10^{6}$ and $4.37 \times 10^{6} \Omega \cdot \mathrm{cm}$ for that with $\mathrm{Cu}$ and $\mathrm{Cr}$ electrodes, respectively (Huang et al., 2019). Such high $R_{C}$ may dominate the $R_{T O T}$ in particular when $L$ is short because the $R_{C H}$ proportionally declines with reducing $L$ while the $R_{C}$ does not. So, the overall device properties can be 
governed by the contact properties (Xu et al., 2017). As a result, largely overestimated mobility may be caused. Only the channel is sufficiently long to ensure the insignificant contact impact so as to extract accurate mobility (Liu et al., 2017). The charge injection is difficult, when the $R_{C}$ is large. So the drain current is suppressed and the electrical characteristics of the device are deteriorated. When the $R_{C}$ is low, the charges, only need a small energy, can pass through the barrier at the interface of electrode and semiconductor. Furthermore, charge accumulation will be quickly and easily occurred for the OFETs with lower $R_{C}$. Therefore, the charge injection is strong, resulting the high performance of IDT-BT OFETs.

\section{Intrinsic Schottky Barrier of Pt- and Au-Based OFETs}

Figures 4A,B show the output characteristics measured at different temperatures $(T)$. According to the thermionic emission, the saturation current of a reverse-biased Schottky junction can be calculated from Eq. (3):

$$
I_{0}=A A^{*} T^{2} \exp \left(-q \phi_{B} / k T\right)
$$

where $A$ is the diode area, $A^{*}$ is the Richardson constant, $T$ is the absolute temperature, $q$ is the elementary charge, $\Phi_{B}$ is the height of the Schottky barrier, and $k$ is the Boltzmann constant (Rhoderick and Williams, 1988). Therefore, through the output characteristics at variable temperatures, $\Phi_{B}$ can be extracted from the plot of $\ln \left(I_{0} / T^{2}\right)$ versus $(q / k T)$. The Pt-based and Au-based devices (measured at $V_{D}=-1 \mathrm{~V}$ and $V_{G}=-$ $50 \mathrm{~V}$ ) clearly show such a dependency (Figures 4C,D). The $\Phi_{B}$ value can be extracted form the slope of the linear fitting of $\ln \left(I_{0} / T^{2}\right)$ versus ( $\left.q / k T\right)$ curve (Xu et al., 2017). Because the imageforce barrier lowering is significant in OFETs, which makes $\Phi_{B}$ highly dependent on the external biases (Bartolomeo et al., 2018), linear fitting of $\Phi_{B}$ is made against $V_{G}$ (at small $V_{D}=$ $1 \mathrm{~V}$ ) to eliminate the associated effect (Brondijk et al., 2012), as seen in Figures 4E,F. The intrinsic Schottky barriers without biasing influence $\left(\Phi_{B, p 0}\right)$ of 0.123 and $0.487 \mathrm{eV}$ are obtained for Pt-based and Au-based devices, respectively. These values are different from the theoretical prediction given by the SchottkyMott limit, due to the different interface chemistry and physics (Xu et al., 2017). The interface of electrode and semiconductor exhibits an additional dipole barrier that tends to change the $W_{F}$ and hence the interface barrier height (Wan et al., 2005). Furthermore, gap states, which can be easily generated in OFETs, are also major influences on Schottky barrier. The hopping ladder and Fermi-level pinning occur and thus the charge injection

\section{REFERENCES}

Bartolomeo, A. D., Grillo, A., Urban, F., Iemmo, L., Giubileo, F., Luongo, G., et al. (2018). Asymmetric schottky contacts in bilayer MoS2 field effect transistors. Adv. Funct. Mater. 28:1800657. doi: 10.1002/adfm.201800657

Brondijk, J. J., Torricelli, F. E., Smits, C. P., Blom, P. W. M., and Leeuw, D. M. (2012). Gate-bias assisted charge injection in organic field-effect transistors. Org. Electron. 13, 1526-1531. doi: 10.1016/j.orgel.2012.04.029 through the Schottky barrier is limited (Tung, 2000). Despite that, one can still draw conclusion. The minor $\Phi_{B, p 0}=0.123 \mathrm{eV}$ means nearly ohmic contacts formed in Pt-based devices, where the charge injection is efficient. By contrast, the high Schottky $\Phi_{B, p 0}=0.487 \mathrm{eV}$ means that the charge injection in Au-based devices is by low-efficiency thermionic emission over high energy barrier. Therefore, the different Schottky barriers lead to different charge injection mechanisms and thus giving rise to very different electrical characteristics.

\section{CONCLUSION}

Schottky-barrier-dependent electrical properties of the transistors using conjugated polymer of IDT-BT and four contact metals have been presented. It is found that the contact's work function changes the Schottky barrier height, alternating the mechanism of charge injection. A highest mobility of $2.79 \mathrm{~cm}^{2} \mathrm{~V}^{-1} \mathrm{~s}^{-1}$ and lowest $R_{C}$ of $1.06 \times 10^{4} \Omega \cdot \mathrm{cm}$ are observed from Pt-based devices, attributable to the nearly ohmic contacts with a small Schottky barrier $\Phi_{B, p 0}=0.123 \mathrm{eV}$. This well explains the greatly improved $I_{O N} / I_{O F F}$ ratio, $V_{T H}$, and SS. Therefore, our results demonstrate that Schottky barrier is crucial for performance optimization to achieve practical organic devices and circuits.

\section{DATA AVAILABILITY STATEMENT}

The datasets generated for this study are available via the corresponding author or other researchers following publication.

\section{AUTHOR CONTRIBUTIONS}

The project was initiated by WL. YX performed the experiments. $\mathrm{YX}, \mathrm{FH}$, and WL prepared the figures and manuscript.

\section{FUNDING}

This work was supported in part by the National Key Research and Development Program of China (Grant No. 2016YFB0501604), the Natural Science Foundation of China (Grant Nos. 61774061 and 61504043), the Natural Science Foundation of Shanghai (Grant No. 19ZR1473400), the NSAF foundation of China (Grant No. U1830130), and the ECNU Academic Innovation Promotion Program for Excellent Doctoral Students (Grant No. YBNLTS2019-028).

Chen, Z., Lee, M. J., Ashraf, R. S., Gu, Y., Seifried, S. A., Nielsen, M. M., et al. (2012). High-performance ambipolar diketopyrrolopyrrole-thieno[3,2b]thiophene copolymer field-effect transistors with balanced hole and electron mobilities. Adv. Mater. 24, 647-652. doi: 10.1002/adma.201102786

Choi, H. H., Rodionov, Y. I., Paterson, A. F., Panidi, J., Saranin, D., Kharlamov, N., et al. (2018). Accurate extraction of charge carrier mobility in 4-probe field-effect transistors. Adv. Funct. Mater. 28:1707105. doi: 10.1002/adfm.20170 7105 
Huang, F. M., Liu, A., Zhu, H. H., Xu, Y., Noh, Y., Li, W. W., et al. (2019). Reliable mobility evaluation of organic field-effect transistors with different contact metals. IEEE Electron Device Lett. 40, 605-608. doi: 10.1109/LED.2019.2901315

Ishii, H., Sugiyama, K., Ito, E., and Seki, K. (1999). Energy level alignment and interfacial electronic structures at organic/metal and organic/organic interfaces. Adv. Mater. 5, 605-625. doi: 10.1002/(SICI)1521-4095(199906)11

Jiang, C., Choi, H. W., Cheng, X., Ma, H., Hasko, D., and Nathan, A. (2019). Printed subthreshold organic transistors operating at high gain and ultralow power. Science 363, 719-723. doi: 10.1126/science.aav7057

Lai, S., Jang, S. K., Cho, J. H., and Lee, S. (2018). Organic field-effect transistors integrated with Ti2CTx electrodes. Nanoscale 10, 5191-5197. doi: 10.1039/ C7NR08677F

Liu, C., Li, G., Pietro, R. D., Huang, J., Noh, Y. Y., Liu, X., et al. (2017). Device physics of contact issues for the overestimation and underestimation of carrier mobility in field-effect transistors. Phys. Rev. Appl. 8:034020. doi: 10.1103/ PhysRevApplied.8.034020

Liu, C., Xu, Y., and Noh, Y. Y. (2015). Contact engineering in organic field-effect transistors. Mater. Today 18, 79-96. doi: 10.1016/j.mattod.2014.08.037

Liu, S. W., Lee, C. C., Tai, H. L., Wen, J. M., Lee, J. H., and Chen, C. T. (2010). In situ electrical characterization of the thickness dependence of organic field-Effect transistors with 1-20 molecular monolayer of pentacene. ACS Appl. Mater. Interfaces 2, 2282-2288. doi: 10.1021/am1003377

Natali, D., and Caironi, M. (2012). Charge injection in solution-processed organic field-effect transistors: physics, models and characterization methods. Adv. Mater. 24, 1357-1387. doi: 10.1002/adma.201104206

Rhoderick, E. H., and Williams, R. (1988). Metal-Semiconductor Contacts. Oxford: Clarendon Press.

Shin, E. Y., Choi, E. Y., and Noh, Y. Y. (2017). Parylene based bilayer flexible gate dielectric layer for top-gated organic field-effect transistors. Org. Electron. 46, 14-21. doi: 10.1016/j.orgel.2017.04.005

Tang, C. G., Ang, M. C., Choo, K. K., Keerthi, V., Tan, J. K., Syafiqah, M. N., et al. (2016). Doped polymer semiconductors with ultrahigh and ultralow work functions for ohmic contacts. Nature 539, 536-540. doi: 10.1038/nature20133

Tung, T. R. (2000). Chemical bonding and Fermi level pinning at metalsemiconductor interfaces. Phys. Rev. Lett. 84, 6078-6081. doi: 10.1103/ PhysRevLett.84.6078
Uemura, T., Rolin, C., Ke, T. H., Fesenko, P., Genoe, J., Heremans, P., et al. (2016). On the extraction of charge carrier mobility in high-mobility organic transistors. Adv. Mater. 28, 151-155. doi: 10.1002/adma.20150 3133

Wan, A., Hwang, J., Amy, F., and Khan, A. (2005). Impact of electrode contamination on the $\alpha-\mathrm{NPD} / \mathrm{Au}$ hole injection barrier. Org. Electron. 6, 47-54. doi: 10.1016/j.orgel.2005.02.003

Wang, R., Guo, Y., Zhang, D., Zhou, H., Zhao, D., and Zhang, Y. (2018). Improved electron transport with reduced contact resistance in n-doped polymer fieldeffect transistors with a dimeric dopant. Macromol Rapid Commun. 39:1700726. doi: 10.1002/marc.201700726

Wang, W. H., Tang, W., Zhao, J. Q., Bao, B., Xing, H., Guo, X. J., et al. (2017). Probing the intrinsic charge transport in indacenodithiophene-cobenzothiadiazole thin films. AIP Adv. 7:125314. doi: 10.1063/1.5001986

Wei, P., Hu, Y., Zhu, Y., Jiang, Y., Feng, X., Li, S., et al. (2018). Dopant/semiconductor/electret trilayer architecture for high-Performance organic field-effect transistors. Adv. Electron. Mater. 4:1800339. doi: 10.1002/ aelm.201800339

Xu, Y., Sun, H. B., Li, W. W., Lin, Y. F., Balestra, F., Noh, Y. Y., et al. (2017). Exploring the charge transport in conjugated polymers. Adv. Mater. 29:1702729. doi: 10.1002/adma.201702729

Yuan, Y. B., Giri, G., Ayzner, A. L., Zoombelt, A. P., Mannsfeld, S. C. B., Chen, J. H., et al. (2014). Ultra-high mobility transparent organic thin film transistors grown by an off-centre spin-coating method. Nat. Commun. 5:3005. doi: 10 . $1038 /$ ncomms 4005

Conflict of Interest: The authors declare that the research was conducted in the absence of any commercial or financial relationships that could be construed as a potential conflict of interest.

Copyright (c) $2020 \mathrm{Xu}$, Huang and Li. This is an open-access article distributed under the terms of the Creative Commons Attribution License (CC BY). The use, distribution or reproduction in other forums is permitted, provided the original author(s) and the copyright owner(s) are credited and that the original publication in this journal is cited, in accordance with accepted academic practice. No use, distribution or reproduction is permitted which does not comply with these terms. 